\title{
CORPUS Corpus
}

Archivos virtuales de la alteridad americana

Vol. 10, No. $2 \mid 2020$

Julio / Diciembre 2020

\section{El Archivo Histórico del Agua del Departamento General de Irrigación de la Provincia de Mendoza, Argentina}

The Historical Archive of Water of the General Department of Irrigation of the Province of Mendoza, Argentina

Facundo Martín, Mark Healey, Juan Pablo Fili, Nicolás Parise and Anabella Engelman

\section{OpenEdition}

Journals

\section{Electronic version}

URL: http://journals.openedition.org/corpusarchivos/3976

DOI: $10.4000 /$ corpusarchivos.3976

ISSN: $1853-8037$

Publisher

Diego Escolar

\section{Electronic reference}

Facundo Martín, Mark Healey, Juan Pablo Fili, Nicolás Parise y Anabella Engelman, « El Archivo Histórico del Agua del Departamento General de Irrigación de la Provincia de Mendoza, Argentina » Corpus [En línea], Vol. 10, №. 2 | 2020, Publicado el 17 diciembre 2020, consultado el 29 diciembre 2020. URL : http://journals.openedition.org/corpusarchivos/3976 ; DOI : https://doi.org/10.4000/ corpusarchivos.3976

This text was automatically generated on 29 December 2020.

Licencia Creative Commons: Atribución-NoComercial 2.5 Argentina (CC BY-NC 2.5 AR) 


\title{
El Archivo Histórico del Agua del Departamento General de Irrigación de la Provincia de Mendoza, Argentina
}

\author{
The Historical Archive of Water of the General Department of Irrigation of the \\ Province of Mendoza, Argentina
}

Facundo Martín, Mark Healey, Juan Pablo Fili, Nicolás Parise and Anabella Engelman

\section{1- Historia institucional y archivística}

1 El archivo que analizamos en este trabajo tuvo su origen en las prácticas burocráticas de administración del agua por parte del Gobierno de Mendoza. En esta provincia andina, el valor económico de la tierra descansa en la posesión de derecho de riego, ya que las lluvias no alcanzan a cubrir el requerimiento mínimo de ningún cultivo. Esta "limitante natural" determinó que las relaciones de propiedad de la tierra estén subordinadas económica y políticamente a la administración del agua. Por tanto, los archivos de esa administración contienen material de gran interés para la historia social, política y ambiental de la provincia.

Desde hace 60 años existe la Biblioteca del Agua en el seno del Departamento General de Irrigación (DGI), con una colección inigualable de libros, revistas, e informes sobre temas del agua en Mendoza. Hace más de diez años que lo dirige Sergio Terrera, quien ha hecho un trabajo formidable de promover su acceso al público. En 2014 empezamos una investigación sobre la historia política del agua a partir de los recursos de la Biblioteca, y con la ayuda de Sergio Terrera, se llegó a hacer una breve indagación en el archivo de material que manejaba el DGI, hasta entonces cerrado a la consulta del público. Tres años después, retomamos el contacto con la Biblioteca del Agua y las autoridades del DGI para realizar un primer acercamiento a la identificación y 
caracterización de los fondos que ahora forman parte del AHA. Así, nos embarcamos en un proceso de trabajo colaborativo entre académicos y personal del DGI que incluyó largas jornadas de trabajo y reflexión, talleres y seminarios. Todo realizado en el marco de las condiciones precarias en cuanto a recursos materiales, tecnológicos y humanos que son habituales en los proyectos de archivística en el país y en la región. A pesar de esto, la necesidad de formalizar la dimensión institucional del archivo fue creciendo y en setiembre de 2018, mediante resolución 909 de Superintendencia, es creado formalmente el Archivo Histórico del Agua bajo la órbita de la División Biblioteca, Editorial y Museo.

IRRIGACIミ́N

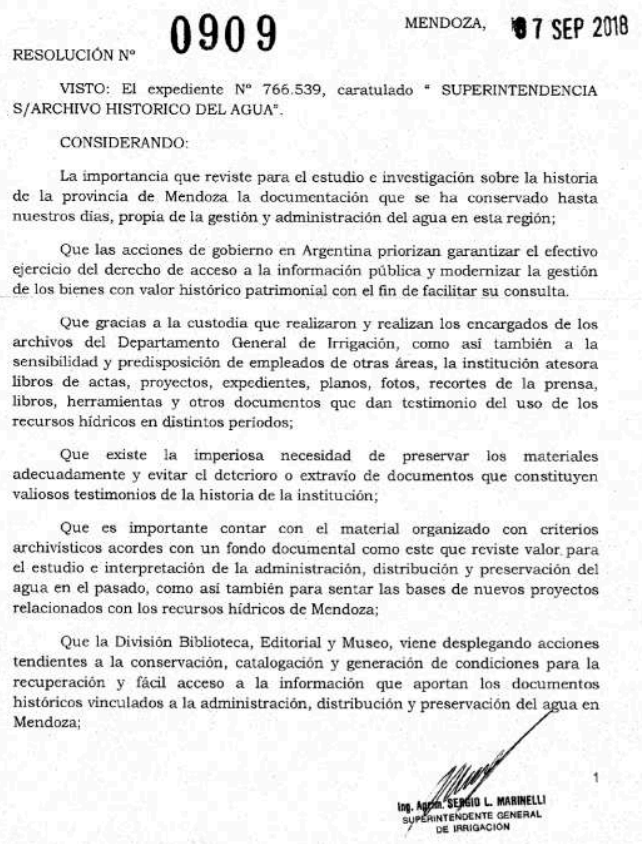

Imagen 1: Resolución de creación del AHA.

Si bien la historia del manejo del agua se remonta a los primeros pobladores de la zona, y el acceso y control del agua son aspectos centrales de la gobernanza colonial española y nacional, las instituciones que generan la documentación del archivo datan de finales del siglo XIX. Para aquellos años, cambios en el manejo legal y material del agua, junto con la llegada del ferrocarril y la inmigración europea, consolidaron la transición hacia una economía vitivinícola y agroindustrial. Así, el agua que escurre de la cordillera de los Andes fue siendo canalizada y administrada progresivamente para la expansión de los oasis irrigados. Hacia 1970 se habían implantado cerca de 300.000 mil hectáreas de viñedos.

El conjunto de la producción documental bajo análisis estuvo determinada por la evolución de la legislación y la institucionalidad hídrica y éstas a la vez "siguieron las huellas de la económica política" (Cano, 1943, p. 49). Durante la época colonial, la administración española desarrolló una forma de gobernar el agua, y después de la independencia, con la extensión del regadío en la geografía provincial, se fueron generando reglamentos particulares acorde a la transcendencia económica de las distintas cuencas. El Reglamento de Policía del Gobierno de Mendoza en 1813 regulaba 
exclusivamente su uso en las ciudades. Hacia 1884 y luego de un período de alta conflictividad, cierta experimentación, y gran dispersión administrativa, se darían las condiciones para un giro trascendente en la administración y, consecuentemente, en la producción documental.

Una vez finalizada la Campaña del Desierto hay un interés por centralizar el manejo del agua, quitándole incumbencia a los municipios. Para afirmar la base de la propiedad privada y una emergente industria vitivinícola, se sanciona en noviembre de 1884 la Ley Provincial de Aguas. Esta dispone un sistema de concesiones de riego a quienes demuestren tierras cultivadas hasta aquel momento. Así, se establecen derechos definitivos para los propietarios de terrenos cultivados mientras que nuevos derechos eventuales serían -en adelante- otorgados pero solo se harían efectivos cuando la oferta del recurso hídrico fuera suficiente para satisfacer la demanda de los primeros. Esto implicaba realizar "aforos" en los ríos de la provincia de manera de registrar los caudales (AR AHA RT cu) y dimensionar el alcance de la distribución. Las concesiones de riego se asentaron por su parte en los padrones generales (AR AHA RT der).\}

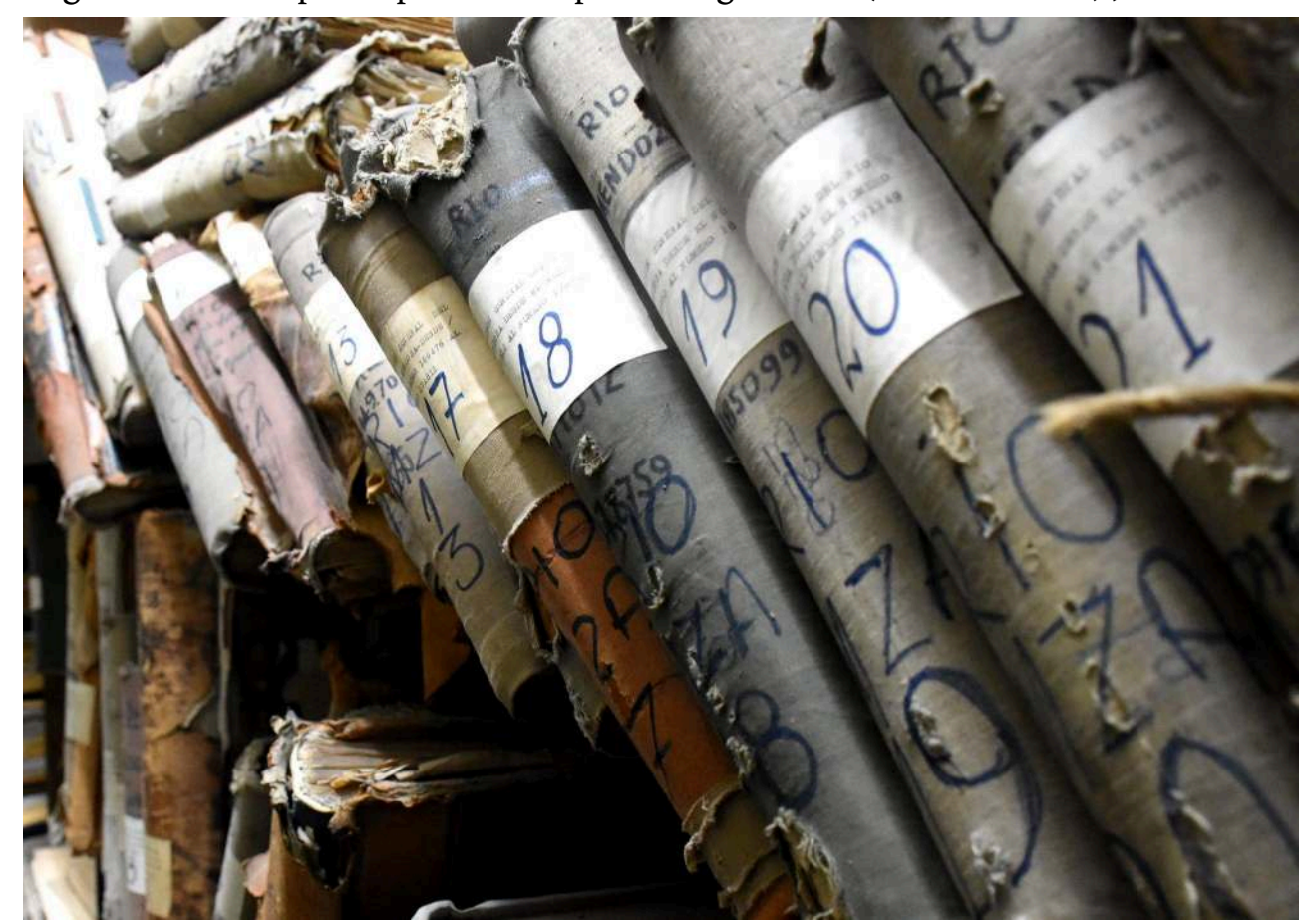

Imagen 2. Padrones Generales. Fuente: AHA-DGI

6 En 1885 se crea por decreto el Departamento General de Aguas, bajo la órbita del Ministerio de Industrias y Obras Públicas (MIOP). Durante la siguiente década, se impulsa la centralización de la administración del recurso a través de la reorganización de las dependencias de topografía, obras públicas e irrigación. La reforma de la constitución provincial de 1894 instituye la figura actual del Departamento General de Irrigación.

7 A partir de entonces se consolida la autonomía administrativa del DGI y, en tanto, su producción documental comienza a estar centralizada y organizada dentro del organismo. Especialmente se destacan las actuaciones del Honorable Consejo de Irrigación, órgano colegiado conformado por 4 vocales representantes de los regantes. Este Consejo sesionará bajo la presidencia del superintendente y atenderá los reclamos de los regantes, los asuntos presupuestarios y de personal, así como lo ateniente a las 
obras hídricas. Las decisiones del Consejo se plasmaron en libros de actas (AR AHA HO caa).

8 La ley 322 de 1905 reorganiza el funcionamiento del DGI sumando a la Superintendencia dos nuevos órganos diferenciados: el Honorable Consejo de Apelaciones (HCA) y el Honorable Tribunal Administrativo (HTA). El Consejo tiene como función revisar las decisiones originales del superintendente, garantizando el cumplimiento de la doble instancia de apelación en la línea administrativa. Por su parte, el Tribunal tiene facultades legislativas (reglamentos, presupuestos) y de control sobre las Inspecciones de Cauce, asociaciones de regantes encargadas de la distribución final del riego. La composición del Consejo es con un representante de cada una de las cuencas administrativas. Por su parte, el Tribunal está conformado por los miembros del Consejo más el superintendente. En lo concerniente a la producción documental, la Superintendencia y el Tribunal emiten resoluciones (AR AHA SUP re y AR AHA HO tar, respectivamente). El Consejo, además de sus propias resoluciones (AR AHA HO car), continuó registrando las sesiones en actas (AR AHA HO caa).

Hacia mediados del siglo XX suceden dos eventos relevantes en términos de la producción y preservación documental del AHA. Por un lado, en 1947 el DGI se traslada a su edificio actual, especialmente construido para tal fin (ver imagen 3). De acuerdo a los testimonios recogidos, durante el traslado se pierde mucha documentación original. Asimismo, se habría producido una ruptura del orden original de parte de la documentación. Por otra parte, en 1959 el archivo administrativo es creado por la resolución 149. La misma nuevamente modifica las funciones de las distintas dependencias del DGI, entre ellas las del hasta entonces archivo general. Posteriormente, en 1963, se crea la Biblioteca Pedro N. Rosas, principal dependencia que anticipó acciones de catalogación y conservación de material documental.

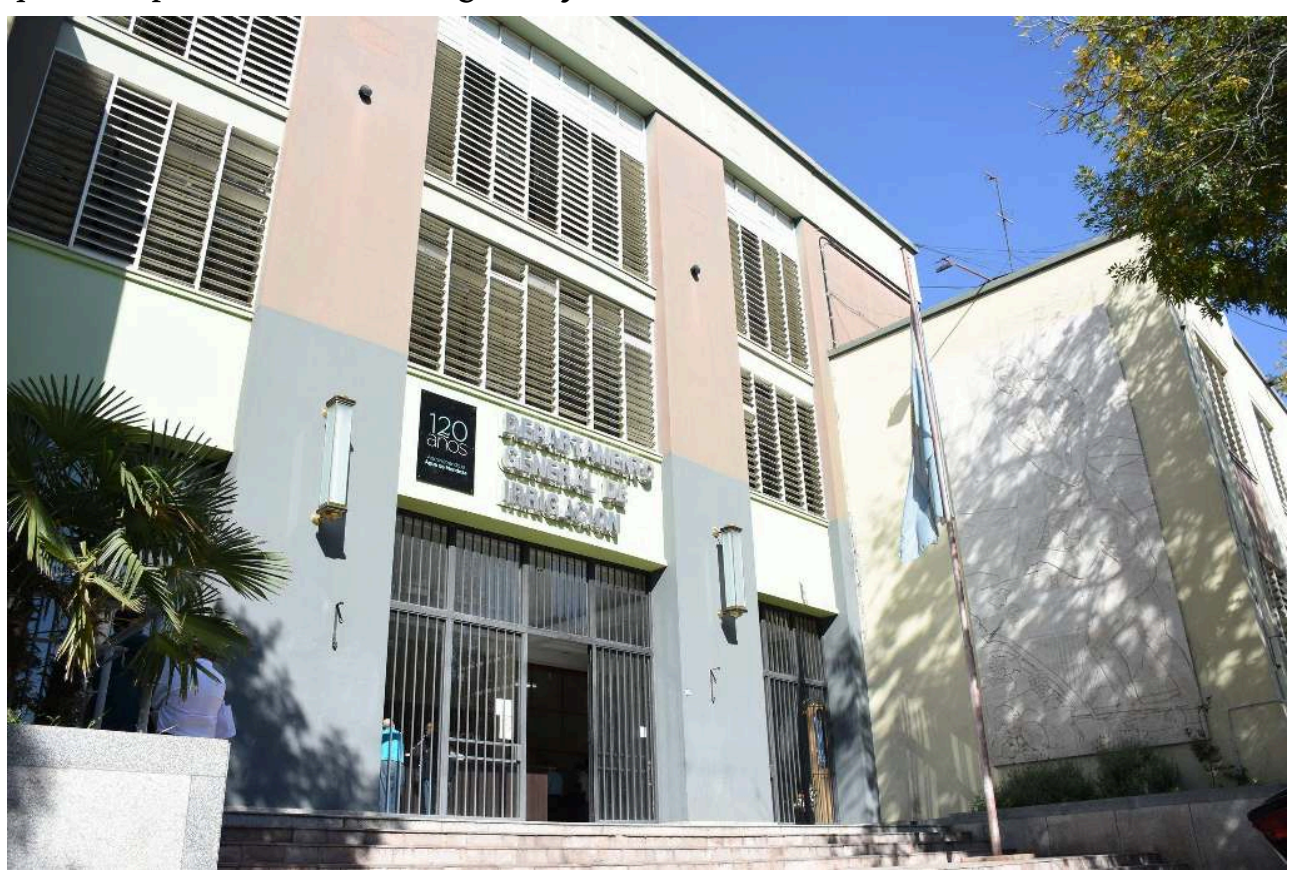

Imagen 3: Edificio actual DGI. Fuente: DGI

Durante la segunda mitad del siglo XX, la producción documental se expande notablemente. Esto está marcado por procesos tales como el crecimiento de los distritos de riego, los conflictos y disputas por el acceso y la distribución, la construcción de 
grandes obras de infraestructura y la administración del agua subterránea. En el plano jurídico y reglamentario, la legislación formal fue siendo reemplazada en la práctica por reglamentación burocrática, provisoria y de carácter excepcional (AR AHA EX dt). Esta creciente complejización de la administración del agua se materializó en nuevos procedimientos administrativos y registros que condujeron a la diversificación del archivo y a la incorporación de voluminosa documentación técnica.

\section{2- ¿Clasificar y desclasificar?}

El inicio del trabajo en el archivo consistió en identificar, dimensionar y diferenciar los fondos y subfondos que lo componían. Al no encontrarse todo el material en un mismo recinto fue necesario visitar diferentes locaciones de la institución. Una vez que se tuvo una idea más completa de las existencias, se construyó una planilla de catalogación que en un primer momento aspiraba a ser exhaustiva. Ese trabajo implicó catalogar un conjunto de documentación elegida al azar para poder dar cuenta, en líneas generales, del tipo de información que contenían. Fue así que nos propusimos realizar un ejercicio de catalogación utilizando los principios de las normas ISAD-G. Lo que aparecía como un ejercicio de aplicación de una serie de criterios estandarizados se transformó en una desafiante experiencia de indagación y reflexión en torno a los archivos. De los diferentes ejercicios realizados, el siguiente cuadro condensa el trabajo realizado hasta el momento:

\begin{tabular}{|l|l|l|c|c|c|c|}
\hline Fondo & \multicolumn{6}{|c|}{ ARCHIVO HISTÓRICO DEL AGUA- DEPARTAMENTO GENERAL } \\
DE IRRIGACIÓN (AR AHA) \\
Fechas extremas: 1855-1960
\end{tabular}

Cuadro 1. Identificación archivística del fondo AHA

En esta descripción general queda a su vez manifiesta la complejidad del acervo documental que aquí se trata. Esta primera división de fondo en algunos casos se subdivide en una o más subdivisiones de fondo, series y subseries. Para realizar esta división del archivo optamos por dos criterios establecidos por la archivística. Por un lado, el productor del fondo y por otro lado la oficina o sección donde se acumulaba. Y esto es así porque la complejidad inherente a la vida de la institución tensó los sentidos otorgados al archivo y sus límites. Eso nos habla de su originalidad y autenticidad ya que todo el tiempo requirió de decisiones sobre la definición del fondo de pertenencia, su procedencia, etcétera. Esto no obsta, como se sabe, para que los sentidos aquí establecidos puedan modificarse en el futuro ya que la irrupción de nuevos datos y 
procesos, como la digitalización, pueden desafiar y complejizar esta primera experiencia.

\begin{tabular}{|c|c|c|}
\hline $\begin{array}{l}1^{\circ} \text { DIVISIÓN } \\
\text { DE FONDO }\end{array}$ & $2^{\circ}$ DIVISIÓN DE FONDO & CÓDIGO ISAD-G \\
\hline \multirow{4}{*}{$\begin{array}{l}\text { ARCHIVO } \\
\text { PASIVO }\end{array}$} & $\begin{array}{c}\text { Expedientes Generales } \\
(1864-1960)\end{array}$ & AR AHA PA exp \\
\hline & MIOP (1904-1960) & AR AHA PA miop \\
\hline & Carpetas suplementarias & AR AHA PA es \\
\hline & Material reservado (1855-1954) & AR AHA PA mr \\
\hline \multirow{6}{*}{$\begin{array}{l}\text { SUPERINTE } \\
\text { NDENCIA }\end{array}$} & Resoluciones (1885-1960) & AR AHA SUP re| \\
\hline & Memorias (1932-1987) & AR AHA SUP me \\
\hline & Presupuesto & AR AHA SUP pp \\
\hline & Digesto & AR AHA SUP do \\
\hline & Difusión (1959-1980) & AR AHA SUP di \\
\hline & Fotos, cartas y contratos & AR AHA SUP fce \\
\hline \multirow{3}{*}{$\begin{array}{c}\text { TRIBUNAL } \\
\text { ADMIN. Y } \\
\text { CONSEJO DE } \\
\text { APELACION } \\
\text { ES }\end{array}$} & Actas HCA (1896-1941) & AR AHA HO caa \\
\hline & Resoluciones HCA (1901-1960) & AR AHA HО car \\
\hline & Resoluciones HTA (1930-1960) & AR AHA HO tar \\
\hline PRENSA & Prensa (1929-2016) & AR AHA PM pr \\
\hline \multirow[t]{3}{*}{ REGISTROS } & Aforos $y$ caudales & AR AHA RT cu \\
\hline & $\begin{array}{c}\text { Libros y Documentos de } \\
\text { Personal (Salarios, Legajos, } \\
\text { Tomeros, etc.) }\end{array}$ & AR AHA RT pr \\
\hline & Derechos /Empadronamiento & AR AHA RT der \\
\hline \multirow{3}{*}{$\begin{array}{l}\text { ESTUDIOS } \\
\text { AUXILIARES }\end{array}$} & Estudios y Proyectos & AR AHA EX ep \\
\hline & Planos & AR AHA EX pl \\
\hline & Dictámenes & AR AHA EX dt \\
\hline
\end{tabular}

Cuadro 2. Cuadro de Clasificación

\section{3- Descripción de las divisiones del fondo}

Las características del fondo del AHA, especialmente su gran volumen, heterogeneidad, y dispersión, hicieron que el trabajo de diseñar y completar los instrumentos de descripción sea de alta complejidad y de una gran demanda de tiempo. De allí también que en esta oportunidad presentamos un primer avance general de los fondos documentales que aloja el AHA. A continuación, ofrecemos una descripción más detallada de las principales divisiones de fondo.

\subsection{Archivo pasivo}

El archivo pasivo del Departamento General de Irrigación se encuentra en el subsuelo de la institución separado del archivo administrativo (activo). Posee cuatro subdivisiones que son: Expedientes Generales, Ministerio de Obras Públicas (MIOP), Carpetas Suplementarias y Material Reservado.

Los Expedientes Generales han sido producidos íntegramente por la institución y sus dependencias internas. Están dispuestos en estanterías donde se ordenan alrededor de 2100 carpetas seriadas que en su interior poseen expedientes numerados. Estos expedientes constituyen el material de mayor volumen y el que más esfuerzos de organización exige dentro del archivo pasivo. Aunque están numerados en secuencia cronológica, carecen de inventario y catalogación. En una revisión (no exhaustiva) de las carpetas más viejas encontramos un expediente del año 1864, el cual sería el más 
antiguo hasta el momento. Según la comprensión del personal del archivo administrativo la existencia del archivo pasivo derivó de la falta de espacio en el primero. En la década del 1990 la documentación más antigua fue trasladada al nuevo espacio (archivo pasivo). El imperativo de preservación de los expedientes responde a posibles solicitudes de los usuarios sobre transferencias, sucesiones, permisos de perforación, entre otros.

Cada carpeta contiene entre 35 y 45 expedientes, dependiendo de la cantidad de fojas que el expediente acumuló. Los temas sobre los que versa se relacionan con reclamos al superintendente por la calidad del servicio o el personal; denuncias de fraude de algún regante; solicitudes de concesiones de riego; reclamos por multas; pedidos por obras o cambios en el riego; pedidos de cumplir con la provisión de agua, así como toda una casuística en torno a formas directas y veladas de obtener derechos de riego. Las crecientes exigencias de la institución fueron creando nuevas instancias de prueba y control que ampliaron considerablemente la cantidad de documentación de un expediente. Por ello, a medida que se avanza en los años se pueden encontrar cada vez más elementos para adjuntar a un caso como mapas, decretos provinciales, cartas de vecinos, copias de boletos de compra y venta de propiedades, etc. Además de mapas catastrales e informes periciales de casos donde la polémica (y el apellido de quien reclamaba) exigían mayor precisión.

Un segundo conjunto lo constituyen los expedientes del Ministerio de Obras Públicas (MIOP) de la Provincia de Mendoza. Este se halla en el mismo recinto que los Expedientes Generales, pero en una pieza contigua. Este agrupamiento de documentos no está organizado. En algunos casos las carpetas se encuentran dispuestas en estanterías y en su mayoría están encimadas y poseen en el lomo un número con la serie que contiene y eventualmente el año. Como fueron producidos fuera de la institución -o antes de que esta existiera en su organización actual- se desconocen todos los avatares de su traslado. A partir del trabajo con dichos expedientes pudimos comprobar que los documentos se relacionan con el Departamento General de Irrigación de manera directa o indirecta. Uno de los puntos fuertes de este conjunto es su clara relación con la obra pública relacionada con el agua para riego $\mathrm{y}$, fundamentalmente, defensa aluvional.

El fondo documental Carpetas Suplementarias está compuesto por grandes acumulados de expedientes abocados en su mayoría a la construcción de grandes obras hidráulicas. Estos se hallan en una habitación contigua al archivo administrativo por lo que su acceso es más restringido que el de las otras divisiones de fondo.

Por último, el conjunto documental de Materiales reservados, se conserva en un armario dentro del archivo administrativo. Está compuesto por un grupo muy heterogéneo y no inventariado de documentos entre los que se destacan libros, cuadernos que compilaban información sobre concesiones, proyectos, folletos, balances y correspondencia. Dicho mueble se ubicaba históricamente en la Superintendencia, pero hace varios años fue trasladado a su nuevo lugar. Esta segunda división de fondo necesita una catalogación pormenorizada antes de comenzar con el proceso de fichaje ISAD-G. 


\section{2- Superintendencia}

20 institución en la oficina del archivo administrativo. El fondo es producido por las resoluciones emitidas por el superintendente de Irrigación. Está compuesto por 402 libros. El primer libro abarca los años 1885-1892 y el último es del año 2008. Todo el fondo se encuentra en estantes, numerados y ordenados del 1 al 402. La mayoría de los libros se encuentran en buen estado, aunque algunos de los más antiguos evidencian signos de humedad en la tapa. Durante la catalogación del fondo pudimos observar que los primeros libros están hechos a mano, y desde el año 1915 en adelante se comienzan a mecanografiar. Es visible en los primeros años que el libro se construía con hojas de los expedientes, por lo cual se explica la ausencia de resoluciones en algunos expedientes generales.

$$
\begin{aligned}
& \text { análisis y atención para no incurrir en errores de información relacionada con los años } \\
& \text { (superposición de años, errores en la nomenclatura de la tapa, etc.). }
\end{aligned}
$$

Cuando sucedió la mudanza de edificio en el año 1947 se venían acumulando expedientes por más de 50 años. Por las resoluciones de Superintendencia podemos saber que el libro dos (que abarca el período 1892-1906) fue encuadernado en la nueva sede, ubicada en la intersección de las calles España y Barcala, en la ciudad de Mendoza. Es decir, que la práctica de ordenamiento y archivo de resoluciones de manera más sistemática se inició en el nuevo edificio. Existen sellos en el libro dos que así lo hacen suponer ya que tienen la leyenda: "Departamento General de Irrigación. España y Barcala".

24 En estos libros se puede encontrar información relativa a presupuestos, pedidos de obras, listado de cauces, control de asistencia del personal, contratos de locación, contrataciones y los antecedentes profesionales del personal y otras temáticas relativas al funcionamiento cotidiano. También se encuentra el decreto del Poder Ejecutivo designando a Laureano Galigniana como primer superintendente.

Las otras tres subdivisiones del fondo (Memoria, Digesto y Presupuesto) tienen por objetivo compendiar información de las acciones y disposiciones acontecidas durante el año de gestión. Estas subdivisiones eran encargadas por Superintendencia y tenían por objetivo facilitar y difundir la información del accionar burocrático de la institución atendiendo a su doble autarquía. Estos tres conjuntos documentales se encuentran a resguardo en la Biblioteca del Agua que también está ubicada en el subsuelo del edificio del DGI.

Un ejemplo del ordenamiento de estos conjuntos documentales lo vemos con las Memorias que son libros confeccionados entre 1932 y 1987. Fueron elaborados usando datos extraídos de las oficinas dependientes de la repartición y posteriormente sometidos a consideración del HCA. Su contenido está referido a los gastos, inversiones y obras realizadas con presupuesto del DGI, por ello se compendiaban en conjunto con los libros de Balance.

27 Los otros dos agrupamientos documentales a los que aludimos arriba: difusión y fotos, cartas y contratos constituyen fondos con material heterogéneo que es necesario 
catalogar antes de poder realizar la ficha archivística correspondiente. Estos fondos se componen de folletería institucional, revistas, propagandas en papel que la institución organizaba para difundir una determinada actividad; por ejemplo, dentro de difusión encontramos las revistas AGUA (1959-1980) que fueron realizadas por la imprenta y editorial de la repartición; consistían en artículos informativos sobre distintas temáticas, cuya autoría pertenecía a los diferentes profesionales que trabajaban en el organismo. También se pueden encontrar cartas, contratos y fotos que han sido donados o han quedado como resabios de alguna actividad dentro del DGI.

\section{3- Honorable Tribunal Administrativo y Honorable Consejo de Apelaciones} elecciones de autoridades de las Inspecciones de Cauce. Se trataban los presupuestos y balances financieros, la proyección y financiación de obras, la gestión y cobranza de impuestos y la determinación de los sueldos de todo el personal. Las actas dan cuenta asimismo de distintas problemáticas que comienza a tener que resolver la institución, como la gestión de solicitudes de permisos para nuevos usos (energía, industria, etc.). temporalmente en las mismas fechas de las sesiones del Consejo. En otros casos dan cuenta de trabajos de investigación previos sobre los archivos (ej. una lista cronológica de los superintendentes). Finalmente, también los libros exponen los conflictos interinstitucionales al implementar la doble autarquía de la institución. Finalmente, son notorios algunos silencios (períodos largos de incluso meses donde presuntamente 
el Consejo no sesionó), tras los cuales luego generalmente se registran cambios de autoridades, como vocales o incluso de la autoridad máxima, el superintendente.

La segunda subdivisión se conforma por las Resoluciones del Honorable Consejo de Apelaciones y está compuesta por 9 libros que abarcan de los años 1901 al 1969. En los libros se encuentra información referida a los presupuestos, los salarios del superintendente y los asesores letrados, etc.

La tercera subdivisión la componen las Resoluciones del Honorable Tribunal Administrativo; si bien el Tribunal comienza a funcionar en 1905, los libros existentes comienzan el 9 de octubre de 1930. Por lo cual, existen versiones de extravío o dispersión de los libros faltantes en las dependencias de la institución. Es posible también que las resoluciones previas estén en los expedientes generales. Este fondo documental está compuesto por 488 libros que abarcan de los años 1930 a 1960.

Los primeros 29 libros de este fondo están escritos a mano, son más grandes que los subsiguientes y en general cada uno abarca una gran cantidad de años que varían según el período. De estos libros algunos poseen índice donde se expresa carátula, folio y fecha. Se ordenan alfabéticamente por inicial de la carátula.

\section{4- Prensa}

Esta primera división de fondo está constituida por 106 libros de prensa que contienen recortes de noticias, entrevistas y artículos de opinión vinculados a la temática del agua en las escalas geográficas local, nacional e internacional. La sistematización y la conservación de los recortes de prensa fueron realizadas en un primer momento por personal de secretaría de Superintendencia. Mientras que, probablemente, los elaborados en los últimos años hayan sido sistematizados por personal del área de prensa del DGI.

Imagen 4. Artículos de prensa. Fuente: AHA-DGI

La colección abarca el período que va desde 1929 hasta 2016, visibilizando los discursos de la prensa que circularon por La Libertad (1929-1957), La Palabra (1935-1954), Los Andes (1929-2016) y otros periódicos carentes de rótulos en los libros. Antes de su incorporación a la Biblioteca del Agua, se hallaba físicamente en un depósito de archivos, con otras fuentes documentales, junto a la playa de estacionamiento, frente a la sede del DGI. La colección fue salvaguardada y posteriormente trasladada para facilitar el acceso y la conservación.

En cuanto a la conservación de los libros de prensa, vale destacar que el estado general de la colección es muy bueno. El material con el que está hecho el papel de prensa de principio de siglo es muy frágil y vulnerable ante la manipulación, y el mínimo roce de las hojas provoca una inmediata ruptura del papel y la consecuente pérdida del valioso material.

Generalmente, los libros poseen rótulos que identifican las fechas de los recortes, aunque no existe una línea de continuidad que denote un criterio común de clasificación de todo el acervo documental. Puede apreciarse que durante algunas gestiones se ha realizado la labor archivística de manera sistemática. Los recortes se hallan adheridos a hojas blancas encuadernadas en libros forrados; algunas de las hojas son lisas con marcas de agua o sellos que identifican a la institución. Otro conjunto de libros lo constituyen carpetas tipo bibliorato $\mathrm{u}$ hojas sueltas anilladas que se almacenan 
dentro de cajas de cartón. Los artículos que se conservaron desde fines del siglo XX, no poseen la técnica de recorte y posterior adherencia a hojas blancas lisas.

41 La labor de producción se inicia en enero de 1929. De acuerdo a nuestra investigación se puede aseverar que el origen del subfondo coincide con la intervención nacional del presidente Yrigoyen sobre el gobierno provincial lencinista de Alejandro Orfila. En el libro titulado Recortes de Diario Los Andes. Empezado el $1^{\circ}$ de enero de 1929, terminado el 20 de diciembre de 1929, un recorte del 3 de febrero documenta la visita del interventor Sr. Borzani a la dependencia para inspeccionarla, siendo recibido por el entonces superintendente Ing. Céspedes. En este libro, también quedó registrado el reordenamiento institucional producido por la intervención nacional en el DGI. Algunos ejemplos de ello son la designación de un nuevo Consejo, la cesantía de empleados y la intervención de la oficina de recaudación.

Los libros de prensa nos permiten dar cuenta de los cambios cotidianos que fueron marcando la historia de la administración del agua tanto a escala institucional como geográfica. Se presume que la intencionalidad de almacenar los libros ha tenido distintas finalidades a lo largo del tiempo, ya que permitieron: a) informar al superintendente y otras autoridades sobre los principales acontecimientos y hechos que transcurrían durante su gestión; b) obtener un panorama general sobre el relato y la visión del agua que era construida por la prensa y otros actores externos con llegada a este tipo de medio; c) que las colecciones sirvieran para comunicar las acciones y logros llevados a cabo durante la gestión de los superintendentes; d) conservar la documentación de prensa como fuente histórica válida para la construcción de las memorias de la institución del agua; e) que los recortes funcionaran como fuente de información en caso de disputas legales.

\subsection{Registros}

La división de fondos Registros posee 3 subdivisiones: Aforos y caudales, Libros y documentos de personal y Derechos/Empadronamientos. En los tres casos se trata de agrupamientos documentales que dan cuenta de las tareas de aforos de los ríos, el manejo de personal de la institución, organización, salarios, etc. y de registro del empadronamiento de los derechos de riego. Se encuentran distribuidos entre el archivo pasivo y el administrativo y son parte fundamental para comprender y cuantificar las dinámicas hacia el interior y exterior del DGI.

\subsection{Estudios Auxiliares}

Por último, la división de fondo Estudios auxiliares está compuesta por tres conjuntos documentales: Planos, Dictámenes, Estudios y proyectos. En los mismos se puede encontrar información referida a estudios encomendados por Irrigación para construcción o modernización de infraestructura hidráulica, aprovechamientos de aguas, estudios de factibilidad y calidad del agua, planos, etc. Del mismo modo se pueden encontrar estudios e informes de abogados y asesores letrados que opinaban y dictaminaban sobre causas presentes en algún expediente. En este caso, la documentación se encuentra distribuida entre el archivo pasivo, asesoría letrada y el departamento de ingeniería de la institución. 


\section{4- Los desafíos en la era digital}

El proyecto de organización y creación del AHA se dio en un momento en que la archivística en particular y las ciencias sociales y humanas en general enfrentan renovados desafíos frente a la transición digital. Rápidamente nos vimos inmersos en la necesidad de incorporarnos a los debates y prácticas que múltiples redes e instituciones vienen traccionando alrededor del mundo. A partir de un trabajo colaborativo entre instituciones académicas y activistas de la cultura libre y las humanidades digitales organizamos talleres con la comunidad local de archivistas para abrir un espacio de diálogo sobre experiencias de digitalización y publicación en línea de fondos públicos. En simultáneo comenzamos un proyecto piloto de digitalización de documentación seleccionada con criterios de conservación y valor documental utilizando un escáner DIY construido por Bibliohack. Desde entonces, se han realizado pruebas con documentación de diferentes formatos (revistas especializadas, actas, libros técnicos, etc.). Esta primera etapa ha permitido consolidar redes de colaboración, así como identificar líneas de acción estratégicas en las que se trabajará en el futuro.
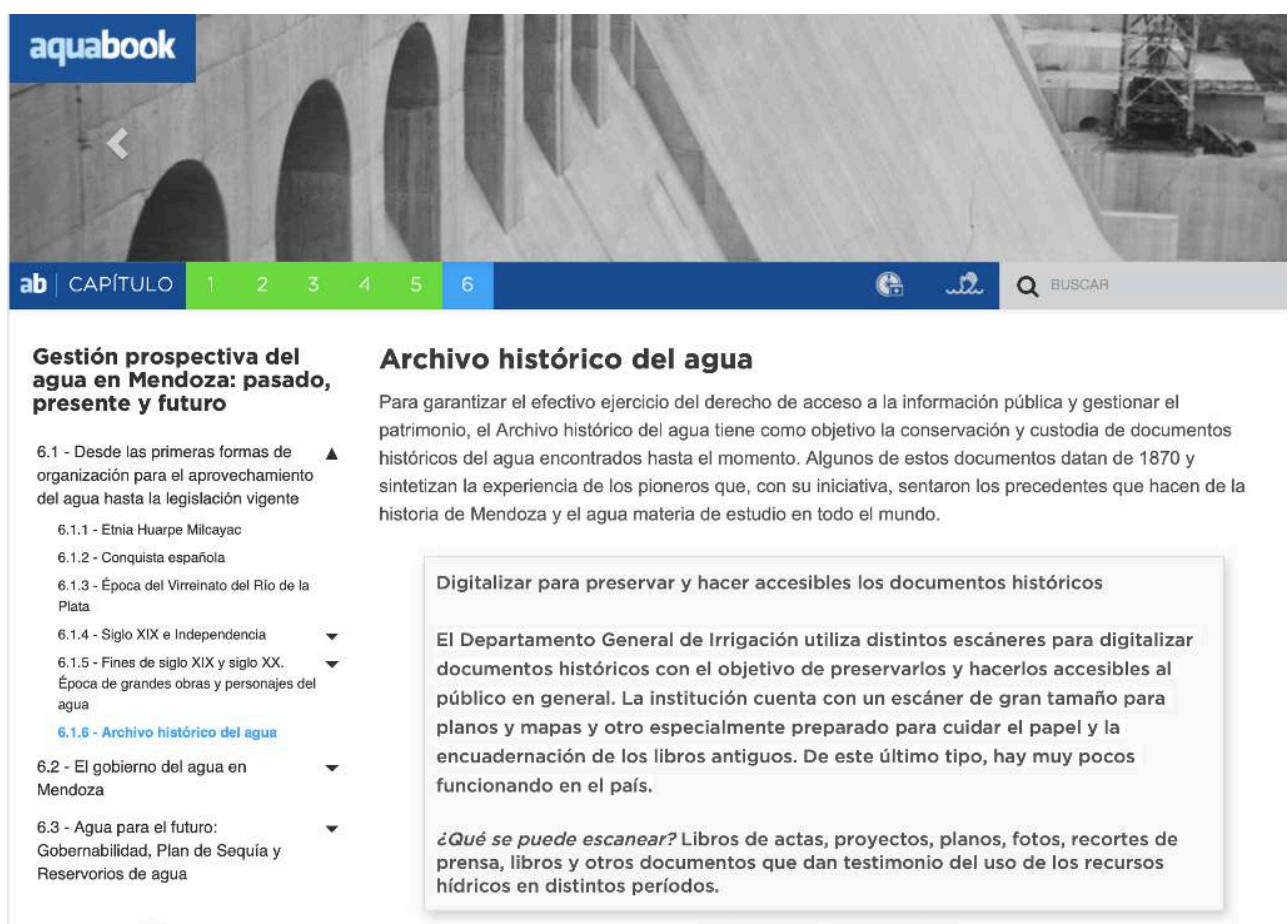

Imagen 5. Aquabook. Fuente: http://aquabook.agua.gob.ar/1079_0. Consultado el 2/4/2020.

\section{5 - Repensando el papel del AHA}

Como todos los archivos, el AHA tiene un sesgo y hasta un ethos que lo rodea, asociado directamente a las estrategias e instituciones que lo fueron organizando. Los documentos bajo análisis, en su mayoría, giran en torno a reclamos sobre el acceso al agua y a la propiedad de la tierra, a la denuncia de "robo" o "malos manejos" del recurso. Se hacen evidentes así un conjunto de reglas de escritura y formas legítimas de reclamar, propias de la formación discursiva de la economía política y del derecho de aguas. Estas políticas del archivo dieron como fruto una producción documental que testimonia claramente el devenir de la gubernamentalización de un Estado provincial. 
Todo el aparato legal e institucional estaba al servicio de la producción de riqueza representada, en este caso, por la necesaria expansión de los oasis irrigados. A la vez, el objeto principal de la institución central era la de atender los reclamos (apelaciones) de los usuarios del riego al tiempo que se registraban y aseguraban sus derechos. Este archivo es testimonio entonces de un caso paradigmático de construcción de autoridad estatal.

Cuando tomamos distancia para poder pensar el archivo, emergen claramente dos aspectos determinantes: por un lado, la monumentalidad de los documentos con los que nos encontramos. En su mayoría son iniciados por propietarios con acceso, conocimiento y manejo de la legislación -a veces para evitarla-. En la historiografía sobre el tema ha predominado, comprensiblemente, un acercamiento galvanizador, deshistorizado e ingenuo a esos documentos -especialmente a los más antiguos y cuyos autores o intervinientes han sido personas públicas- $-{ }^{1}$ Sabemos que los documentos no son nunca transparentes ni reflejos inmediatos de la realidad que se pretende reconstruir. Antes bien son registros producidos intencionalmente y luego hallados y preservados por otras instituciones o personas. Asociado a este primer elemento, durante mucho tiempo la documentación más antigua, que ahora forma parte del AHA, ha estado cubierta por el extenso manto de la secrecía que predominó y predomina en muchos archivos estatales. Esto ha condicionado la producción historiográfica hasta el momento y abre ahora nuevos y desafiantes escenarios de investigación y reflexión crítica.

Estas características se relacionan con el hecho de que la documentación fue producida en el marco de procesos administrativos y, en algunos casos, conteniendo información "sensible". Estos dos aspectos influyeron notablemente en el estado y características de los fondos que hoy son parte del AHA. Sin duda otra fuente de "inaccesibilidad" fue la falta de clasificación y organización del material o la falta de procedimientos claros para utilizar los índices existentes más allá de su uso administrativo o interno. Actualmente, en el marco de la formalización del AHA existen procedimientos establecidos y voluntad institucional de ampliar y garantizar la accesibilidad en el marco de los estándares internacionales.

El estado de conservación de la documentación es muy variable. Encontramos fondos muy bien conservados, como las resoluciones y actas del HTA, y otros con gravísimos problemas de conservación y alto grado de deterioro, como algunas resoluciones de Superintendencia más antiguas. Además, las condiciones edilicias del espacio físico, particularmente del archivo pasivo, distaban de ser las ideales para la correcta conservación documental. A partir de los inicios de los trabajos de inventariado, desde la coordinación del AHA se han realizado ingentes esfuerzos para adecuar las instalaciones y la infraestructura. Al mismo tiempo se ha concretado la relocalización de algunos documentos con el fin de detener los procesos de deterioro.

50 La concepción contemporánea de la archivística se articula a partir de la triple función social de los archivos como fuente de investigación histórica, preservación de la memoria colectiva y promoción de derechos. En este sentido hay múltiples desafíos por delante frente a la consolidación del AHA.

51 En términos de agenda de investigación, una visión renovada y aguda acerca de los diversos mecanismos que se utilizaron para otorgar o restringir derechos de agua y sus efectos económicos, políticos y sociales constituye una pregunta central. En el mismo sentido, indagar sobre las formas de construcción de autoridad estatal asociadas al 
manejo del agua desde una mirada histórica y espacial aparece como una línea de reflexión promisoria (Fili y Engelman, 2020). Finalmente, una evaluación cuidadosa de las intrincadas relaciones entre la planificación estatal y el papel del conocimiento técnico puesto a su servicio pueden ofrecer lecciones no despreciables para el presente. Un desafío mayor está constituido por las formas y vías teóricas y metodológicas para dar cuenta de la experiencia humana a partir de lo que el archivo no dice, lo que calla en la repetición. Se trata entonces de hacerle preguntas al archivo que excedan y desestabilicen las respuestas enunciadas en sus formatos predeterminados. Se trata también de trazar una investigación acerca de las decisiones del archivo, de hacer visibles las zonas grises de manera tal de reconstruir los vacíos producidos por las políticas oficiales de archivo. Otros actores y usuarios, mucho más diversos que los convencionales, deben entrar en la conversación. En tiempos de crisis ambientales y cambio global acelerado, que el AHA pueda asumir una pedagogía pública rigurosa y democrática constituye una enorme oportunidad.

\section{Agradecimientos}

Queremos agradecer al personal del Departamento General de Irrigación y especialmente a Marcela Andino, Sergio Terrera, Mariela Irusta, Miguel Quiroga y Alejandro Cruz, quienes, en contextos sumamente adversos para el trabajo archivístico, no solo hacen posible sino que promueven de forma entusiasta cada una de las acciones que se desarrollan en torno al Archivo Histórico del Agua.

\section{BIBLIOGRAPHY}

Cano, G. (1943). Estudios de Derechos de Aguas. Mendoza: Editor Valerio Abeledo.

Fili, J. P. y Engelman, A. A. (2020). El dominio público y privado de las aguas del río Tunuyán: Litigios a finales del siglo XIX en Mendoza (Argentina). Anuario de Historia Regional y de las Fronteras, 25(1), 47-68.

\section{NOTES}

1. Es común encontrar entre los elencos del Departamento General de Irrigación a la mayoría de los apellidos que formaban los círculos de la oligarquía provincial. Pasar por el cargo de superintendente era a la vez una de las vías posibles de acceso a la gobernación. 


\section{ABSTRACTS}

This article presents the Historical Water Archive of the General Irrigation Department of the provincial government of Mendoza, Argentina. The article begins with the historical development of the institutions producing the archive, then surveys the main collections it contains, and sketches the challenges and opportunities of un-covering historical sources barely visible or analyzed until the present.

En este artículo presentamos el Archivo Histórico del Agua (AHA) del Departamento General de Irrigación perteneciente a la Provincia de Mendoza. Para esto realizamos un recorrido por la historia de las instituciones productoras de los archivos, caracterizamos los principales fondos hallados y delineamos los desafíos y oportunidades que implica el des-cubrimiento de fuentes históricas poco visibles o no analizadas hasta el momento.

\section{INDEX}

Keywords: Mendoza, Water, Rights, Property, Irrigation, Archive

Palabras claves: Mendoza, Agua, Derechos, Propiedad, Riego, Archivo

\section{AUTHORS}

\section{FACUNDO MARTÍN}

Universidad Nacional de Cuyo y Consejo Nacional de Investigaciones Científicas y Técnicas, Argentina

Correo electrónico: fdmartingarcia@gmail.com

\section{MARK HEALEY}

University of Connecticut, EEUU

Correo electrónico: mark.healey@uconn.edu

\section{JUAN PABLO FILI}

Consejo Nacional de Investigaciones Científicas y Técnicas, Argentina Correo electrónico: juanpablo_fili@yahoo.com

\section{NICOLÁS PARISE}

Consejo Nacional de Investigaciones Científicas y Técnicas, Argentina Correo electrónico: nicolasparise.s@gmail.com

\section{ANABELLA ENGELMAN}

Consejo Nacional de Investigaciones Científicas y Técnicas, Argentina Correo electrónico: anabellaengelman@gmail.com 